\title{
DETERMINACIÓN BIOMÉTRICA DE LAS CARACTERÍSTICAS MORFO-FACIALES DE LOS ADULTOS JÓVENES EN SANTANDER
}

\author{
Andrés Enrique Fajardo Cuartas \\ Odontólogo, U. Santo Tomás, Estudiante de III Año Especialización en Ortodoncia, U. Santo Tomás.
}

Autor responsable de correspondencia: Andrés Enrique Fajardo $C$.

Dirección de correo electrónico: andresfajardo@hotmail.com

\begin{abstract}
RESUMEN
objetivo: Determinar las características morfológicas faciales de tejidos blandos en la población de estudiantes de la Universidad Santo Tomás de Bucaramanga para el año 2004-2005.

Materiales y métodos: Se realizó un estudio transversali tipo descriptivo, tomando una muestra de 85 adultos jóvenes, con un nivel de significancia del 95\%, un poder del 80\% y comparación de proporciones con una diferencia del 10\%. A cada uno de ellos se le aplicó una encuesta, se incluyeron variables sociodemográficas, medidas horizontales faciales como ancho cigomático, ancho gonial, distancia interalar; medidas verticales faciales como altura facial, tercio superior, medio e inferior y medidas angulares de perfil como ángulo nasolabial y convejidad. Se tomaron los puntos antropométricos y se realizaron las mediciones previamente descritas directamente en el paciente y en fotografias. Se codificaron y sistematizaron en formato Epi-Info 6.04. La información se analizó mediante el software STATA 6.0, a través de la prueba de "t" de student de varianzas homogéneas y el análisis de varianza de un factor para comparar más de dos grupos; con un nivel de significancia inferior a 0.05 .

Resultados: Se observó una media correspondiente al ancho cigomático, ancho gonial, altura facial, subnasal-stomionl y stomion2-menton, mayor en los hombres que en las mujeres con significancia estadística ( $p<0,0000)$; sin embargo se observó un comportamiento contrario en stomion1-stomion2, en donde fue mayor en las mujeres que en los hombres con una significancia estadística de $(\mathrm{p}<0.0068)$.

Conclusión: Existe un dimorfismo marcado en esta población en la mayoría de las mediciones y se amerita el uso de promedios santandereanos para las medidas como ancho cigomático, gonial, bucal, base nasal, altura facial y ángulo frontonasal. [Fajardo AE. Determinación biométrica de las características morfofaciales de los aduitos jóvenes en Santander. Ustasalud Odontología 2006; 5:101 - 106]
\end{abstract}

Palabras clave: Antropometría facial, Biometría.

\section{BIOMETRIC DETERMINATION OF MORPHOFACIAL CHARATHERISTICS IN YOUNG ADULTOS IN SANTANDER}

\begin{abstract}
Objective: To determine the morphofacial characteristics of soft tissues in a population of students of the Santo Tomas University for the year 2004-2005.

Materials and methods: The observational cross sectional study was done, taking a sample of 85 young adults. The sociodemographic variables were included, facial horizontal mecsures as wide cigomatic, wide gonial, distances interalar; facial vertical measures as facial height, superior and inferior half and angular measures of profile like angle, nasolabial angle and convexity. The measures were done directly in the patient and in their pictures. They were coded and they systematized in format EPI-INF0 6.04. The information was analyzed by means of the software STATA 6.0, with the $t$ of student of homogeneous variances and the analysis of variance of a factor to compare more than two groups, with a level of inferior significance at 0.05 .

Results: A stocking corresponding to the cigomatic wide, gonial wide, facial height, subnasal-stomion1 and stomion2-chin, were observed bigger in the men than in the women with statistical significance ( $<<0,0000)$; however a contrary behavior was observed in stomion1-stomion2 where was bigger in the women than in the men with a statistical significance of ( $<<0.0068$ ). Conclusion: Exists a marked dimorfism in this population in most of the measurements. Therefore, it will be important to use these averages for the measures as wide cigomatico, gonial, buccal, nasal base, facial height and frontonasal angle frontonasal
\end{abstract}

Key words: Facial anthropometry, Biometry.

Recibido para publicación: 10 de ciiciembre de 2006. Aceptado para publicación: 13 de febrero de 2007. 


\section{INTRODUCCIÓN}

La armonía facial en el ser humano, tiene una gran influencia en el proceso de aceptación ante la sociedad y en el bienestar del individuo como tal. ${ }^{1,2}$

Todas las culturas han tenido sus propias ideas alrededor de la estética; los egipcios, romanos y griegos, establecieron cánones de anatomía armoniosa y proporciones ideales que están de acuerdo con el concepto moderno de ortognatismo. ${ }^{3}$

Con la aparición de Angle el padre de la ortodoncia moderna y posteriormente con Tweed, la Ortodoncia puso como una de sus metas el logro de una buena armonía facial y oclusal. ${ }^{4.5}$

Hasta la fecha se ha realizado algunos estudios sobre la estética facial, sin tener en cuenta la oclusión dental, ${ }^{1,2}$ Holdaway demostró que dadas las variaciones en espesor, altura y tono muscular de los tejidos blandos, era necesario estudiar su contorno, y no solamente las características óseas, como es usual en la antropologia. ${ }^{2}$

Downs estableció que los individuos con salud oral óptima, balance funcional y estética, tendían a tener ciertas características comunes en el perfil y que el espesor del mentón era primordial en la evaluación. ${ }^{6}$

Hasta los año 70, las diferencias según el sexo no habían sido estudiadas, ni mucho menos respaldadas con un análisis de tipo estadístico; $C o x$ encontró que las medidas verticales faciales masculinas y femeninas para efectos de comparación, se expresaban mejor en el porcentaje de altura facial total; ${ }^{7}$ Ruskin demostró que había una diferencia significativa en la apreciación estética de los perfiles de acuerdo con el sexo; ${ }^{1}$ Spradley y otros encontraron que dichas diferencias dependían del grupo étnico, la edad promedio y la selección de la muestra; por otra parte, Park y Burstone observaron que la variación en la protrusión labial podía explicarse por la diferencia en la convexidad de la cara entre ambos sexos. ${ }^{8}$

Giraldo y Rubio realizaron una apreciación cefalométrica del perfil de tejidos blandos y compararon los resultados con el patrón americano, encontrando diferencias estadísticamente significativas en varias medidas, como ángulo nasolabial y ángulo de la convexidad.

El propósito de esta investigación fue determinar las características morfológicas faciales de tejidos blandos en la población de estudiantes de la Universidad Santo Tomás de Bucaramanga, comparándola a su vez con los promedios internacionales de normalidad de mayor aceptación, para de esta forma lograr una evaluación mas ajustada a las características de la región santandereana, no solo simplificando el análisis, sino que permitiendo un diagnóstico más acertado de los pacientes.

\section{MATERIALES Y MÉTODOS}

Se realizó un estudio transversal tipo descriptivo

El universo fueron los adultos jóvenes matriculados en la Universidad Santo Tomás de Bucaramanga durante el año 2004 y 2005. La prevalencia de la anormalidad de las morfologías faciales es de una $40 \%$. Se calculó una muestra de 85 adultos jóvenes, con un nivel de significancia del $95 \%$, un poder del $80 \%$ y comparación de proporciones con una diferencia del $10 \%$.

Dentro de los criterios de inclusión que se consideraron fueron, adulto joven entre 18 a 29 años de edad sin considerar género, con padres y abuelos nacidos en el territorio santandereano, oclusión Clase I de Angle, balance facial visible, y firma del Consentimiento Informado por parte de cada uno de ellos. Dentro de los criterios de exclusión estaban, adulto joven con antecedentes médicos de secuelas oro-faciales., ortodoncia u ortopedia previa y anodoncia congénita de laterales o dos o más dientes vecinos ausentes.

Inicialmente, se realizó una prueba piloto en diez sujetos, con el fin de establecer los procedimientos de campo mas adecuados, probar los instrumentos de recolección, verificar la utilidad del examinador como elementos de recolección de datos y estimar tiempos y rendimientos para principales procedimientos de estudio.

Se consideraron variables sociodemográficas como género, edad y como variables clínicas ancho cigomático, ancho gonial, distancia intercantal, distancia pupilar mesofacial, ancho de base nasal, ancho bucal, altura facial, tercio superior, tercio medio, tercio inferior, subnasal-stomion1, stomion2-menton, canto interno-canto externo, distancia interalar, que tuvieron un nivel de medición razón continua y su codificación fue en milimetros. Las variables ángulo nasolabial, ángulo frontonasal y ángulo de la convexidad, tienen un nivel de medición razón continua y su codificación fue en grados.

Se tomaron los puntos antropométricos y se realizaron las mediciones frontales previamente descritas directamente en el paciente, con la ayuda de un calibrador milimetrado marca SOMET y las medidas angulares, se realizaron con la ayuda de una regla cefalométrica (protractor) marca Ortho-Organizers, en fotografias de perfil tomadas con una cámara digital $\mathrm{Ca}$ non A-70 3.2 megapixeles.

Estos datos fueron registrados en los instrumentos diseñados preestablecidos en el protocolo. Después se digitaron los datos recolectados en una base de datos previamente elaborada en Epi - Info $6.04 d .{ }^{10}$ La base de datos fue digitada por duplicado por dos personas diferentes y cruzadas entre sí para detectar errores de digitación. Previo a cualquier manipulación, se grabó la base de datos original del estudio. Cada error fue corregido, quedando registro de los cambios realizados.

102 
Un programa de computador: Validate de Epi - Info 6.04d fue usado para comparar cada dato en dos archivos resultantes con el fin de verificar la identidad de la información.10

Se realizó un análisis descriptivo del cuestionario de información general y de las medidas morfológicas faciales obteniendo medidas de tendencia central y de dispersión; ya que las variables cuantitativas cumplieron con los criterios de una distribución normal, para las variables de interés de cada grupo recolectadas durante el desarrollo del estudio con sus correspondientes intervalos de confianza (IC) 95\%. La información se analizó mediante el software STATA 6.0. Las pruebas estadísticas fueron: la prueba de " $t$ " de student de varianzas homogéneas y el análisis de varianza de un factor para comparar más de dos grupos; con un nivel de significancia inferior a 0.05 .

\section{RESULTADOS}

En los resultados del estudio para los 85 personas se encontró que la distribución por sexo fue de 37 hombres (44\%); la media de la edad era de 25.47 ( + : 3.34) para toda la población y, en los hombres fue de 24.2 (+: 3.06 ) y en las mujeres de 27.08 (+:3.03).
Se observó una media correspondiente al ancho cigomático mayor en los hombres que en las mujeres con significancia estadisti$\mathrm{ca}(\mathrm{p}<0,0000)$; de igual manera con la media correspondiente al ancho gonial. En las otras medidas como distancia intercantal, canto interno-canto externo, pupilar mesofacial, ancho bucal, base nasal, distancia interalar, no se observó diferencias estadísticamente significativas como se aprecia en la Tabla 1.

Con respecto a las medidas de tipo vertical, la altura facial arrojó una media mayor en los hombres que en las mujeres, con una significancia $(\mathrm{p}<0,0000)$; de igual manera se comportaron el tercio inferior, subnasal-stomion1 y stomion2-menton. Sin embargo se observó un comportamiento contrario en stomion1stomion2, en donde fue mayor en las mujeres que en los hombres con una significancia estadística de $(\mathrm{p}<0.0068)$. En las otras variables de medición vertical, no se encontró diferencias estadísticamente significativas (Tabla 2).

En las medidas angulares de perfil, en el ángulo frontonasal se observó una media mayor en las mujeres que en los hombres, con una significancia de $(\mathrm{p}<0.0066)$. Pero en las variables ángulo nasolabial y ángulo de la convexidad no hubo diferencias estadísticamente significativas (Tabla 3).

Tabla 1. Comparación por género, medidas horizontales frontales en tejidos blandos.

\begin{tabular}{|c|c|c|c|c|c|}
\hline & \multicolumn{2}{|c|}{ HOMBRES } & \multicolumn{2}{|c|}{ MUJERES } & \multirow{3}{*}{$\mathbf{P}^{*}$} \\
\hline & \multicolumn{2}{|c|}{$\mathrm{n}=37$} & \multicolumn{2}{|c|}{$n=48$} & \\
\hline & Media & D. E. & Media & D. E. & \\
\hline Ancho zigomático & 141,71620 & 4,6060666 & 133,39580 & 4,90002 & 0,0000 \\
\hline Ancho gonial & 121,81080 & 16,141450 & 117,0000 & 16,52851 & 0,001 \\
\hline Distancia intercantal & 35,01351 & 2,148922 & 34,85417 & 2,93721 & 0,7821 \\
\hline Distancia pupilar mesofacial & 34,29730 & 2,234389 & 33,72917 & 2,56191 & 0,3222 \\
\hline Base nasal & 34,98649 & 1,952514 & 34,32292 & 1,96645 & 0,1230 \\
\hline Ancho bucal & 56,29730 & 1,753803 & 55,42708 & 2,44077 & 0,0988 \\
\hline Distancia intercantal & 35,79730 & 1,596262 & 34,90625 & 2,53834 & 0,2630 \\
\hline Distancia interalar & 39,63514 & 3,449333 & 40,68750 & 4,37671 & 0,1158 \\
\hline
\end{tabular}

* prueba t de Student D. E. Desviación estándar n = población del grupo

Tabla 2. Comparación por género, medidas verticales frontales en tejidos blandos.

\begin{tabular}{|c|c|c|c|c|c|}
\hline & \multicolumn{2}{|c|}{ HOMBRES } & \multicolumn{2}{|c|}{ MUJERES } & \multirow{3}{*}{$\mathbf{P}^{*}$} \\
\hline & \multicolumn{2}{|c|}{$n=37$} & \multicolumn{2}{|c|}{$n=48$} & \\
\hline & Media & D.E. & Media & D. E. & \\
\hline Altura facial & 134,02700 & 4,552113 & 128,16670 & $7,458,83$ & 0,0000 \\
\hline Tercio superior & 56,14865 & 6,147340 & 56,52083 & 5,35938 & 0,5984 \\
\hline Tercio medio & 64,21622 & 6,078812 & 62,22917 & 6,04677 & 0,2896 \\
\hline Tercio inferior & 69,24324 & 4,855726 & 65,70833 & 2,93880 & 0,0006 \\
\hline Subnasal stomion 1 & 22,02703 & 3,218751 & 19,04167 & 2,90237 & 0,0000 \\
\hline Stomion 2-menton & 46,67568 & 2,934981 & 45,16667 & 2,76246 & 0,0136 \\
\hline Stomion 1 - stomion 2 & 0,45946 & 0,767195 & 1,52083 & 1,75025 & 0,0068 \\
\hline
\end{tabular}

Tabla 3. Comparación por género, medidas angulares de perfil en tejidos blandos.

\begin{tabular}{|c|c|c|c|c|c|}
\hline & \multicolumn{2}{|c|}{ HOMBRES } & \multicolumn{2}{|c|}{ MUJERES } & \multirow{3}{*}{$\mathbf{P}^{*}$} \\
\hline & \multicolumn{2}{|c|}{$n=37$} & \multicolumn{2}{|c|}{$n=48$} & \\
\hline & Media & D. E. & Media & D. E. & \\
\hline Ángulo nasolabial & 97,10811 & 7,813037 & 98,41667 & 1,00591 & 0,6718 \\
\hline Ángulo frontonasal & 132,75680 & 11,353920 & 139,31250 & 7,32863 & 0,0066 \\
\hline Ángulo convejidad & 99,45946 & 3,274022 & 11,10417 & 3,49004 & 0,1256 \\
\hline
\end{tabular}

" prueba t de Student, D.E. Desviación estándar, $\mathrm{n}=$ población del grupo 
Al comparar las mediciones del estudio con las aceptadas internacionalmente, se encontraron en los hombres variables por encima de estos rangos, como lo fueron el ancho zigomático, el ancho gonial, la distancia intercantal, la distancia pupilar mesofacial, el ancho bucal y la altura facial. Sin embargo, el tercio inferior y el ángulo de la convejidad tuvieron un comportamiento diferente, siendo menores en este caso (Tabla 4).

Finalmente, al relacionar las mediciones con los rangos internacionales, se encontraron en las mujeres variables por encima de estas mediciones, como lo fueron el ancho zigomático, el ancho gonial, la distancia intercantal, la distancia pupilar mesofacial, el ancho bucal, base nasal, la altura facial y el ángulo frontonasal. El resto de mediciones coincidieron con los rangos aceptados mundialmente (Tabla 5).

En la tabla 6 se muestran las características morfológicas faciales en tejidos blandos para la población de estudiantes de la Universidad Santo Tomás.

Tabla 4. Comparación de referencia facial para hombres con medias internacionales aceptadas.

\begin{tabular}{|c|c|c|c|c|c|}
\hline & \multicolumn{4}{|c|}{ HOMBRES (n = 37) } & \multirow{3}{*}{$\mathbf{P}^{*}$} \\
\hline & \multicolumn{2}{|c|}{ Santander } & \multicolumn{2}{|c|}{ Internacional } & \\
\hline & Media & D. E. & Media & D. E. & \\
\hline Ancho zigomático & 141.71 & 4.60 & 137 & 4.3 & 0,0000 \\
\hline Ancho gonial & 121,81 & 16,14 & 97 & 5,8 & 0,0000 \\
\hline Distancia intercantal & 35,01 & 2.14 & 33 & 2,7 & 0,0007 \\
\hline Distancia pupilar mesofacial & 34,20 & 2,23 & 33 & 2 & 0,0104 \\
\hline Ancho bucal & 56,29 & 1,75 & 53 & 3,3 & 0,0000 \\
\hline Altura facial & 134,02 & 4,75 & 121 & 6,8 & 0,0000 \\
\hline Tercio inferior & 69,24 & 4,85 & 72 & 6 & 0,0331 \\
\hline Ángulo convejidad & 9,94 & 3,27 & 12 & 4 & 0,0182 \\
\hline
\end{tabular}

* pruebat de Student D. E. Desviación estándar $n=$ población del grupo

Tabla 5. Comparación de referencia facial para mujeres con medias internacionales aceptadas.

\begin{tabular}{|c|c|c|c|c|c|}
\hline & \multicolumn{4}{|c|}{ MUJERES (n = 48) } & \multirow{3}{*}{$\mathbf{P}^{*}$} \\
\hline & \multicolumn{2}{|c|}{ Santander } & \multicolumn{2}{|c|}{ Internacional } & \\
\hline & Media & D. E. & Media & D. E. & \\
\hline Ancho zigomático & 133,39 & 4,0 & 130 & 5,3 & 0,0016 \\
\hline Ancho gonial & 117,0 & 16,5 & 91 & 5,9 & 0,0000 \\
\hline Distancia intercantal & 34,85 & 2,93 & 32 & 2,4 & 0,0000 \\
\hline Distancia pupilar mesofacial & 33,72 & 2,56 & 31 & 1,8 & 0,0000 \\
\hline Base nasal & 34,32 & 1,96 & 31 & 1,9 & 0,0000 \\
\hline Ancho bucal & 55,42 & 2,44 & 50 & 3,2 & 0,0000 \\
\hline Altura facial & 128,15 & 7,45 & 112 & 5,2 & 0,0000 \\
\hline Ángulo frontonasal & 139,31 & 7,32 & 134 & 1,8 & 0,0000 \\
\hline
\end{tabular}

* pruebat de Student D.E. Desviación estándar $\mathrm{n}=$ población del grupo

Tabla 6. Características morfológicas faciales en tejidos blandos para la población de estudiantes de la Universidad Santo Tomás.

\begin{tabular}{|c|c|c|c|c|}
\hline & \multicolumn{2}{|c|}{ HOMBRES } & \multicolumn{2}{|c|}{ MUJERES } \\
\hline & Media & Intervalo de Confianza & Media & Intervalo de Confianza \\
\hline Ancho zigomático & 141,7 & $140,1-143,2$ & 133,3 & $131,9-134,8$ \\
\hline Ancho gonial & 121,8 & $126,4-127,1$ & 119,0 & $117,4-120,6$ \\
\hline Ancho intercantal & 35,01 & $34,2-35,7$ & 34,8 & $34,0-35,7$ \\
\hline Distancia pupilar mesofacial & 34,2 & $33,5-35,04$ & 33,7 & $32,9-34,4$ \\
\hline Base nasal & 34,9 & $34,3-35,6$ & 34,3 & $33,7-34,8$ \\
\hline Ancho bucal & 56,2 & $55,7-56,8$ & 55,4 & $54,7-56,1$ \\
\hline Altura facial & 134 & $132,4-135,6$ & 128,1 & $126-130,3$ \\
\hline Tercio superior & 56,1 & $54,09-58,19$ & 56,5 & $45,9-58,07$ \\
\hline Tercio medio & 64,2 & $62,1-66,2$ & 62,2 & $60,4-63,0$ \\
\hline Tercio inferior & 69,2 & $67,6-70,8$ & 65,7 & $64,8-66,5$ \\
\hline Subnasal - stomion 1 & 22,02 & $20,9-23,1$ & 19,04 & $18,1-19,8$ \\
\hline Stomion 2 -- menton & 46,6 & $45,6-47,6$ & 45,1 & $44,3-45,0$ \\
\hline Stomion 1 - stomion 2 & 0,4 & $0,2-0,7$ & 1,52 & $1,01-2,02$ \\
\hline Distancia canto interno conto externo & 35,7 & $35,2-36,3$ & 34,9 & $34,1-35,6$ \\
\hline Distancia interalar & 39,6 & $38,4-40,7$ & 40,6 & $39,4-41,9$ \\
\hline Ángulo nasolabial & 97,01 & $94,5-99,7$ & 98,4 & $95,4-101,3$ \\
\hline Ángulo frontonasal & 132,7 & $128,9-136,5$ & 139,3 & $137,1-141,4$ \\
\hline Ángulo convejidad & 9,9 & $8,8-11,0$ & 11,1 & $10,09-12,1$ \\
\hline
\end{tabular}

104

Ustasalud Odontología 2006; 5:101 - 106

Fajardo AE. 


\section{DISCUSIÓN}

De un grupo de 85 personas valoradas, de las cuales 48 eran mujeres (66\%) y 37 hombres (44\%), los resultados del estudio arrojaron medidas superiores en los hombres que en las mujeres en la mayoría de los casos, lo cual implicaría un dimorfismo sexual, teniendo similitud con los datos obtenidos por Echeverri y Mejia en 1989 y por Amado y colaboradores. ${ }^{12,13}$

Farkas y Munro, en 1987,i aseguraron que en promedio, los valores correspondientes al ancho facial, como lo son el ancho cigomático, el ancho gonial, son proporcionalmente mayor en los hombres que en las mujeres; resultado similar a este estudio.9 Sin embargo las medidas como distancia intercantal, base nasal y ancho bucal, a pesar de ser superiores en los hombres, no mostraron diferencias estadísticamente significativas.

Con respecto a las medidas de tipo vertical, en donde se analizaron la altura facial y la altura del tercio medio, superior e inferior, se observó que el tercio medio fue el que tuvo un comportamiento similar tanto en hombres como en mujeres, tal como lo aseguraron Echeverri y Mejia en $1989 .{ }^{12}$

Lo anterior se puede explicar como lo asegura Farkas, ${ }^{9}$ que debido a que el maxilar superior tiene un crecimiento vertical menor que el mandibular y más rápido, entonces de esta forma el tercio medio es el que menos cambia durante el desarrollo craneofacial.

Pero en cuanto al tercio inferior, el cual tuvo una diferencia significativa siendo superior en los hombres, se podría concluir como lo hizo Hagg, ${ }^{14}$ que es debido a que la mandíbula puede presentar un crecimiento residual, que es mas marcado en el sexo masculino; si embargo en un estudio realizado por jiménez en la población de Belmira - Antioquia, (raza negra), se observó un aumento considerable en el tercio medio e inferior especialmente en las mujeres, en comparación con individuos de Damasco - Antioquia, (raza mestiza), concluyendo que estos pueden ser debidos a patrones genéticos o raciales patognomónicos de la raza negra. ${ }^{15}$

Como complemento al análisis del tercio inferior se puede agregar, basándose en un estudio realizado por Zagarra, en 1981,16 en el cual se buscaba encontrar diferencias en la población colombiana entre indios cubeos, mestizos e individuos descendientes de raza caucásica, se encontró que existía diferencia entre el grupo indígena y los otros dos restantes, mientras que al comparar los mestizos y los caucásicos no se encontraron diferencias significativas, que se asemejaban en un patrón retrusivo craneofacial y en una altura facial mayor que la del indígena; y si lo anterior se relaciona con los datos obtenidos en la población santandereana, los resultados son similares y respondería a que en esta zona del país hubo alta influencia europea en la época de la conquista.

La única medida vertical que tuvo un comportamiento diferente a todas, fue stomion1 - stomion2, en donde fue superior en las mujeres que en los hombres tal como lo describió Jones y Betancur y comparándola con los datos de Burstone y Park los cuales muestran una similitud en los valores entre ambos sexos, ${ }^{17,8}$ se podría mencionar que la población femenina colombiana e incluso la santanderena tiene una tendencia a presentar proquelia superior e inferior, que asociada a un tercio inferior aumentado, lo que podría dar lugar a una brecha interlabial.

Por otro lado, en cuanto a las medidas angulares, se observó que en este estudio, el ángulo nasolabial, que a pesar que tuvo unos valores menores que los establecidos por Farkas ${ }^{9}$ estaban cercanos a los parámetros de normalidad; sin embargo, al compararlos con los datos del estudio antioqueño de Jones y colaboradores, en el cual el valor fue mucho menor, ${ }^{17}$ entonces esta diferencia amplia dentro de un mismo país se podría relacionar con la tendencia de la población de Antioquia a presentar un tercio medio reducido y más retruido comparado con el tercio inferior.

Finalmente, es importante hacer énfasis que en Colombia se han realizado otros estudios de crecimiento y desarrollo craneofacial, tales como los de Amado y colaboradores, ${ }^{13}$ Cárdenas y colaboradores, ${ }^{18}$ y Montoya y colaboradores, ${ }^{19}$ y los anteriormente mencionados, en donde se demuestran diferencias marcadas de las características faciales de otras poblaciones mundiales; es factible que en cada zona o región de Colombia, se sigan encontrando quizás algunas similitudes, pero muchas mas diferencias entre ellas mismas, consecuencia de la variada migración europea, lo cual llevó a un complejo y variado patrón facial de mestizaje.

\section{CONCLUSIONES}

Existe dimorfismo sexual en la población santandereana, siendo mayor en los hombres en las variables: ancho cigomático, ancho gonial, ancho bucal, altura facial, tercio inferior, subnasalstomion1, stomion2-menton, y mayor en las mujeres en las variables: stomion1-stomion2 y ángulo frontonasal.

Se pueden aplicar en la población santandereana los promedios aceptados internacionalmente para las siguientes variables:

- Base nasal igual a distancia pupilar mesofacial, a distancia intercantal y a distancia canto interno-canto externo.

- Subnasal-stomion1.

Tercio superior y tercio medio.

La siguientes medidas ameritan el uso de los promedios santandereanos:

- Ancho cigomático, genial y bucal.

Base nasal.

- Altura facial.

- Angulo frontonasal. 


\section{BIBLIOGRAFÍA}

1. Lines PA, Lines RR, Lines CA. Profilometrics and facial esthetics. Am J Orthod 1978; 73: 648 - 657 .

2. Holdaway RA. A soft tissue cephalometric analysis and its use in orthodontic treatment planning. Part I. Am J Orthod 1983; 84: 1 - 28.

3. Merrifield L. The profile line as an aid in critically evaluating facial esthetics. Am J Orthod 1966; 52: $804-822$.

4. Snodell SF, Nanda RS, Currier GF: A longitudinal cephalometric study of transverse and vertical craniofacial growth. Am J Orthod Dentofac Orthop 1993; 104: 471 - 483.

5. Spradley FL, Jacobs JD, Crowe DP. Assessment of the anteroposterior soft-tissue contour of the lower facial third in the ideal young adult. Am J Orthod 1981; 79: 316 - 325.

6. Giraldo E, Rubio G. Apreciación cefalométrica del perfil de tejidos blandos en adultos jóvenes colombianos. Universitas Odontológica 1992; 11: $23-30$.

7. Cox NH, van der Linden FP. Facial harmony. Am J Orthod 1971; 60: $175-183$.

8. Park Y, Burstone C. Soft tissue profile fallacies of hard-tissue standards in treatment planning. Am J Orthod. 1986; 90: 52-62

9. Farkas L. Munro I. Anthropometric Facial Proportions in Medicine. Charles C. Thomas Publisher, Springfield, Illinois USA, 1987

10. CDC Atlanta, 1999. EPI-INF0 6.04d.

11. Stata Corporation, 2002. Stata 8.0

12. Echeverri JI, Mejia JF, Jiménez IV. Crecimiento y desarrollo facial y general de individuos del Valle de Aburra. CES Odontología 1990; 3: $21-26$.

13. Amado J, Chavarriaga M, Estrada A, Uribe L. Anólisis cefalométrico en niños con dentición mixta entre 8 y 12 años de edad del Valle de Aburrá. Medellín, 1988.

14. Hagg V, Taranger J. Maduration Indicators and the pubertal growth sport. Am J Orthod 1982; 82: 217-230.

15. García JM, Guzman JC, Tobón OM, Jiménez ID. Estudio comparativo de crecimiento y desarrollo general y craneofacial en dos poblaciones diferentes (Belmira y Damasco). CES Odontología 1993; 6: 33 - 40

16. Zagarra, J. A comparative cephalometric study between the craneofacial patterns of the Colombian mestizo, native indian and caucassian European ancestry. The Pittsburg University, Penn., 1981.

17. Bojanini A, Betancur J, Jones M. Análisis biométrico de las características faciales de la población colombiana.

18. Cárdenas G, López O, Lema O, Espinal F, Echeverri L, Vélez H. Estudio cefalométrico de 310 niños en una comunidad desnutrida. Ant Med. 1969; 19: 5- 20.

19. Montoya JA, Naranjo JM, Vargas JM, Jiménez ID. Análisis de crecimiento y desarrollo craneofacial y general en una muestra de individuos de la población de Damasco. CES Odontología 1992; 5: 45- 51.

\section{Especialización en Odontopediatría 170556170616800441100}

\section{ODONTOLOGIAA INFANTIL}

Servicios de Odontopediatría y Ortodoncia Preventiva CALIDAD A BAJO COSTO

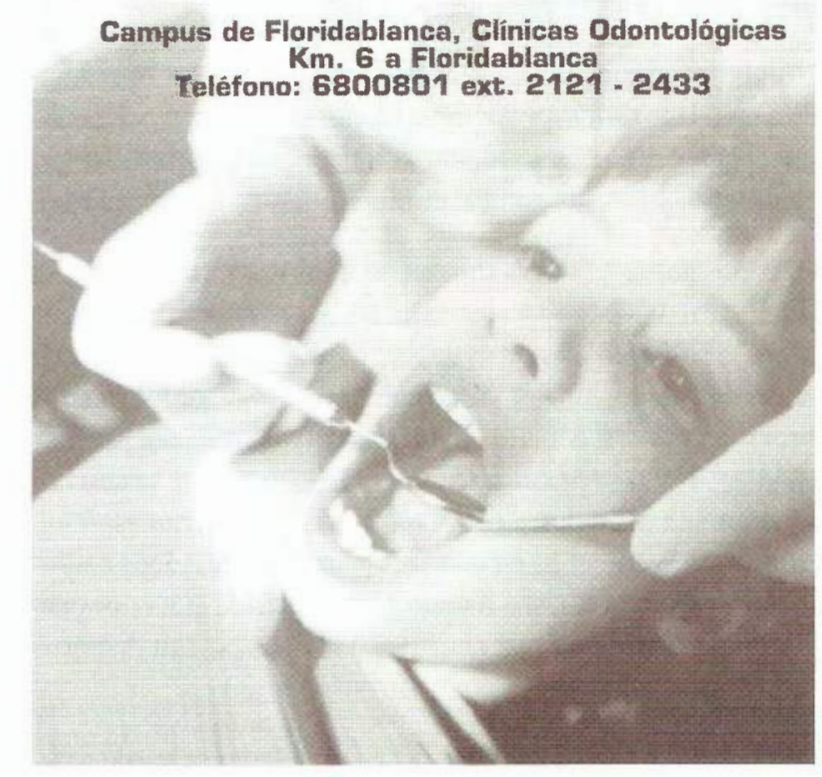

106

Ustasalud Odontología 2006; 5:101 - 106

Fajardo $A E$. 